hep-th/0502118

\title{
Mirror effect induced by the dilaton field on the Hawking radiation
}

\author{
Kengo Maeda* and Takashi Okamura ${ }^{\dagger}$ \\ * Department of General Education, Kobe City College of Technology \\ 8-3 Gakuen-higashi-machi, Nishi-ku, Kobe 651-2194, Japan \\ $\dagger$ Department of Physics, Kwansei Gakuin University, Sanda 669-1337, Japan
}

We discuss the string creation in the near-extremal NS1 black string solution. The string creation is described by an effective field equation derived from a fundamental string action coupled to the dilaton field in a conformally invariant manner. In the non-critical string model the dilaton field causes a timelike mirror surface outside the horizon when the size of the black string is comparable to the Planck scale. Since the fundamental strings are reflected by the mirror surface, the negative energy flux does not propagate across the surface. This means that the evaporation stops just before the naked singularity of the extremal black string appears even though the surface gravity is non-zero in the extremal limit.

\footnotetext{
*e-mail:kmaeda@kobe-kosen.ac.jp

${ }^{\dagger}$ e-mail:okamura@ksc.kwansei.ac.jp
} 


\section{INTRODUCTION}

It is generically agreed that quantum gravity might remove pathological objects in classical general relativity like singularity. One of the difficulties in quantizing gravity is the non-renormalizability of itself. So, we expect that string theory is the best of candidates for consistent quantum gravity theory since string theory is free of the ultraviolet divergences. It was shown that the first-quantized string is well defined on orbifold and weak curvature singularities $[1,2]$. However, every curvature singularity is not resolved at the first-quantization level (See ref. [2]) since mass of the first-quantized string gets excited infinitely for strong curvature singularities. Such a problem could be resolved by constructing string field theory describing pair creation of strings.

Lawrence and Martinec [3] derived an effective string field equation from the fundamental string action in which the coupling term $\alpha^{\prime} R \Phi$ between curvature of the world-sheet $R$ and the dilaton field $\Phi$ is absent. Based on the equation the pair creation rate was calculated on various cosmological models [3-5]. When the dilaton field is almost constant, this coupling term is negligibly small as this is $O\left(\alpha^{\prime}\right)$. The coupling term, however, could be dominant near singularity where the dilaton field diverges. In this paper we investigate the effect of the dilaton field on the string creation in near-extremal black strings. The effective field equation describing the string creation is derived from a string action conformally coupled to the dilaton field. To simplify the equation, we dimensionally reduce the fundamental string to a particle by omitting the string excitations and by compactification.

In Einstein Gravity charged black holes are described by Reissner-Nordström solution, where the temperature approaches zero in the extremal limit. So, the extremal black hole in Einstein Gravity is stable against the Hawking radiation. In string theory there are black branes which have finite temperature and a singular horizon in the extremal limit. This leads physicists to imagine that the singular horizon, or naked singularity appears by evaporation. This expectation was partially verified in [6] by investigating dilatonic black holes.

We investigate the evaporation process of five-dimensional NS1 black string with Kaluza-Klein charge (W-charge) by the string creation. When the W-charge is zero, the temperature of the black string becomes infinite in the extremal limit and hence the quasi-adiabatic approximation breaks down. When the W-charge is non-zero, the temperature is non-zero but finite in the limit. So, we can test whether the naked singularity appears within the quasi-adiabatic approximation.

For the non-critical string model, we find that the effective field equation becomes singular at a timelike surface outside the horizon when the black string is comparable to the string scale $\sqrt{\alpha^{\prime}}$. 
We show that the effective field equation has a unique solution for the initial data with finite energy so that the natural function space of initial data is the Sobolev space $H^{1}$. The well-posedness of massive scalar field was shown in some interesting spacetimes for the field in the Sobolev space [7]. Since the string current passing through the timelike surface is zero for each mode in the Sobolev space, this surface plays a role of a mirror surface. So, the negative energy flux does not propagate across the surface, implying that the evaporation by the string creation stops before the occurrence of the naked singularity. For the critical string model, we find that the effective field equation does not become singular until the naked singularity appears, implying that the black string continues to evaporate. In Sec.IV, we will argue that the occurrence of the naked singularity could be prevented if we consider the time dependence of the dilaton field.

For completeness, we also investigate the stability of near-extremal NS1 black string in which temperature is zero in the extremal limit. In general it is possible for the zero temperature black hole to evaporate by the spontaneous discharge process, as shown in [8]. We show that the evaporation continues unless the $\mathrm{W}$-charge vanishes. Since the mass-loss rate is smaller than the charge-loss rate, it leaves the extremal state. So, the naked singularity does not occur.

This paper is organized as follows. In the next section, we formulate string creation based on the particle picture. In section III, we discuss the evaporation process and superradiance by the string creation. Section IV is devoted to conclusion and discussions.

\section{FORMULATION OF STRING CREATION BASED ON PARTICLE PICTURE}

We study the string creation problem in the particle picture, which is obtained by dimensionally reducing the string action with the dilaton coupling term $\alpha^{\prime} R \Phi$ to the particle one. If we dimensionally reduce the action with the coupling term $\alpha^{\prime} R \Phi$ directly, then we are faced with a bit technical difficulty. It comes from the breaking of the Weyl invariance by the coupling term $\alpha^{\prime} R \Phi$ at the classical level. Indeed, in the conformal gauge $h_{a b}=e^{2 \chi} \eta_{a b}$, the coupling term produces

$$
\alpha^{\prime} \int d^{2} \sigma \sqrt{-h} R \Phi=2 \alpha^{\prime} \int d^{2} \sigma(\partial \Phi) \cdot(\partial \chi) .
$$

The interaction term shows that the field $\chi$ to be unphysical gauge mode becomes dynamical degrees of freedom. Of course, the reason is that we do not incorporate quantum corrections. In order to overcome this difficulty by incorporating the quantum corrections, we translate the Polyakov-type action with $\alpha^{\prime} R \Phi$ term into the action with Weyl invariant dilaton coupling term which gives the same partition function as the original Polyakov-type action. In the appendix A, we explain it in detail. 
The Lorentzian action of a test string propagating in $D$-dimensional target space, which corresponds to the Euclidean action (A.13) is given by

$$
\begin{aligned}
& \mathcal{S}=\frac{-1}{4 \pi \alpha^{\prime}} \int d^{2} \sigma \sqrt{-h}\left(h^{a b} \tilde{G}_{M N}(X)+\epsilon^{a b} B_{M N}(X)\right) \partial_{a} X^{M} \partial_{b} X^{N}, \\
& \tilde{G}_{M N}(X):=G_{M N}(X)+\gamma \alpha^{\prime} \partial_{M} \Phi(X) \cdot \partial_{N} \Phi(X),
\end{aligned}
$$

where $\gamma=6 /\left(D-D_{\text {crit }}+1\right)$ and we drop "hat" from the fiducial metric and use the capital Latin letters as the indices of the target space.

\section{A. Kaluza-Klein reduction of the test string}

Hereafter, we would like to consider a test string in $(d+1)$-dimensional spacetimes obtained by toroidal compactification. And then, we assume that the $(d+1)$-dimensional spacetimes have one Killing direction and a test string extends along the direction only.

Concretely, we consider the background fields as follows;

$$
\begin{aligned}
& d s^{2}=G_{M N} d X^{M} d X^{N}=d s_{d+1}^{2}+\sum_{M=d+1}^{D-1}\left(d X^{M}\right)^{2}, \\
& d s_{d+1}^{2}=g_{\mu \nu}(x) d x^{\mu} d x^{\nu}+k^{2}(x)\left(d X^{d}+\hat{A}_{\mu}(x) d x^{\mu}\right)^{2}, \\
& \boldsymbol{B}=\mathcal{A}_{\mu}(x) d x^{\mu} \wedge d X^{d}, \quad \Phi=\Phi(x),
\end{aligned}
$$

where $\mu, \nu=0,1, \cdots, d-1$, and then the "effective metric" $\tilde{G}_{M N}$ becomes

$$
\tilde{G}_{\mu \nu}=\tilde{g}_{\mu \nu}(x):=g_{\mu \nu}(x)+\gamma \alpha^{\prime} \partial_{\mu} \Phi(x) \cdot \partial_{\nu} \Phi(x), \quad \tilde{G}_{d d}=G_{d d}=k^{2}(x),
$$

and so on. In the target spacetime with its metric (II.5), we would like to investigate the dynamics of the the test string which extends along the $X^{d}$-axis,

$$
x^{\mu}=z^{\mu}(\tau), \quad X^{d}=-m \sigma+y(\tau), \quad X^{M}=\text { fixed } \quad(M=d+1, \cdots, D-1) .
$$

From the canonical form $P_{M} \dot{X}^{M}=p_{\mu} \dot{z}^{\mu}+P_{d} \dot{y}$, we know that $P_{d}$ is the canonically conjugate momentum of $y$. Furthermore, the variable $y$ becomes cyclic coordinate, since $X^{d}$ is the Killing direction.

In the ansatz, we can take the gauge conditions for the world sheet reparametrization,

$$
h_{00}=-\mathcal{N}^{2}(\tau) e^{2 \chi(\tau)}, \quad h_{11}=e^{2 \chi(\tau)}, \quad h_{01}=0,
$$

and we can obtain the effective action for the Kaluza-Klein (KK) compactified test string,

$$
\mathcal{S}=\kappa \int d \tau\left(\frac{1}{2 \mathcal{N}} \tilde{g}_{\mu \nu} \dot{z}^{\mu} \dot{z}^{\nu}+\frac{k^{2}}{2 \mathcal{N}}\left(\dot{y}+\hat{A}_{\mu} \dot{z}^{\mu}\right)^{2}+m \mathcal{A}_{\mu} \dot{z}^{\mu}-\mathcal{N} \frac{m^{2} k^{2}}{2}\right)
$$


where $\kappa:=l / 2 \pi \alpha^{\prime}$ and we take $0 \leq \sigma \leq l$.

Since the variable $y$ is cyclic coordinate, the canonically conjugate momentum of $y$ is a constant $p_{y}=\left(\kappa k^{2} / \mathcal{N}\right)\left(\dot{y}+\hat{A}_{\mu} \dot{z}^{\mu}\right)=: q$, and then we can simplify the action (II.10) with the Rauth function,

$$
\mathcal{S}^{\prime}=\int d \tau\left[\frac{\kappa}{2 \mathcal{N}} \tilde{g}_{\mu \nu} \dot{z}^{\mu} \dot{z}^{\nu}+\mathbb{A}_{\mu}(z) \dot{z}^{\mu}-\frac{\mathcal{N}}{2 \kappa} V(z)\right],
$$

where

$$
\mathbb{A}_{\mu}(x):=(\kappa m) \mathcal{A}_{\mu}(x)+q \hat{A}_{\mu}(x), \quad V(x):=(\kappa m)^{2} k^{2}(x)+\left(\frac{q}{k(x)}\right)^{2} .
$$

From the former equation in Eq.(II.12), we know that this KK-compactified test string carries the Kalb-Ramond charge $\kappa m$ and the W-charge $q$.

\section{B. Quantization}

Hereafter, we denote the string position coordinates by $x$. In order to quantize the system, we should make Hamiltonian formulation. Following the standard procedure, we have a Hamiltonian constraint

$$
\mathcal{H}^{\prime}:=\frac{1}{2 \kappa}\left[\left(p_{\mu}-\mathbb{A}_{\mu}\right)\left(\tilde{g}^{-1}\right)^{\mu \nu}\left(p_{\nu}-\mathbb{A}_{\nu}\right)+V(x)\right] \approx 0
$$

where $\left(\tilde{g}^{-1}\right)^{\mu \nu}$ is the inverse matrix of $\tilde{g}_{\mu \nu}$.

According to the standard procedure of the canonical quantization, we take a wave function $\Psi(x)$ and replace the momenta with the derivatives, and then, we obtain the equation of motion,

$$
0=2 \kappa \mathcal{H}^{\prime} \Psi(x)=-\left[\mathcal{D}_{\mu}\left(\tilde{g}^{-1}\right)^{\mu \nu} \mathcal{D}_{\nu}-V(x)\right] \Psi(x), \quad \mathcal{D}_{\mu}:=\nabla_{\mu}-i \mathbb{A}_{\mu},
$$

where we adopt the covariant derivative with respect to the original metric $g_{\mu \nu}$ as momentum operators, $p_{\mu} \rightarrow-i \nabla_{\mu}$ and adopt the operator ordering by which there exists the conserved inner product. Indeed, for the current $J^{\mu}$ defined by

$$
J^{\mu}\left(\Psi_{1}, \Psi_{2}\right):=i\left(\tilde{g}^{-1}\right)^{\mu \nu}\left[\Psi_{1}^{*} \mathcal{D}_{\nu} \Psi_{2}-\left(\mathcal{D}_{\nu} \Psi_{1}\right)^{*} \Psi_{2}\right],
$$

the equation $\nabla_{\mu} J^{\mu}=0$ holds and we can define the conserved inner product as

$$
\left(\Psi_{1}, \Psi_{2}\right):=\int_{\Sigma} d \Sigma n_{\mu} J^{\mu}\left(\Psi_{1}, \Psi_{2}\right)=i \int_{\Sigma} d \Sigma n_{\mu}\left(\tilde{g}^{-1}\right)^{\mu \nu}\left[\Psi_{1}^{*} \mathcal{D}_{\nu} \Psi_{2}-\left(\mathcal{D}_{\nu} \Psi_{1}\right)^{*} \Psi_{2}\right] .
$$

The equation (II.14) is also derived from the action

$$
S_{\Psi}=-\int d^{d} x \sqrt{-g}\left[\left(\tilde{g}^{-1}\right)^{\mu \nu}\left(\mathcal{D}_{\mu} \Psi\right)^{*}\left(\mathcal{D}_{\nu} \Psi\right)+V(x)|\Psi|^{2}\right] .
$$


By regarding the field $\Psi(x)$ as the quantized field operator and using the inner product (II.16), we could formally make the second quantization and argue the "string creation" in the same manner as the particle creation.

Hereafter, we are interested in static cases as

$$
g_{\mu \nu} d x^{\mu} d x^{\nu}=-N^{2} d t^{2}+h_{i j} d x^{i} d x^{j}, \quad \mathbb{A}_{\mu} d x^{\mu}=\mathbb{A}_{t} d t, \quad \nabla_{\mu} \Phi=D_{\mu} \Phi,
$$

where $D_{\mu}$ is the covariant derivative with respect to the spatial metric $h_{\mu \nu}$. In this case, the field equation (II.14) becomes

$$
\mathcal{D}_{t}^{2} \Psi=-K \Psi, \quad K:=-N\left[D_{i}\left(N\left(\tilde{h}^{-1}\right)^{i j} D_{j}\right)-N V\right]
$$

and then, the inner product (II.16) suggests a natural inner product for functions on the spatial hypersurface $\Sigma$ as

$$
\langle u, v\rangle:=\int_{\Sigma} \frac{d \Sigma}{N} u^{*} v
$$

For later convenience, we introduce another inner product on the spatial surface, the Sobolev one

$$
\langle u, v\rangle_{\mathrm{Sob}}:=q^{2} \int d \Sigma \frac{u^{*} v}{N}+\int d \Sigma N\left[\left(\tilde{h}^{-1}\right)^{i j}\left(D_{i} u\right)^{*}\left(D_{j} v\right)+V u^{*} v\right]
$$

where $q$ is some real constant. We note that the operator $K$ is hermitian with respect to not only the inner product (II.20), but also the Sobolev inner product,

$$
\begin{aligned}
& \langle u, K v\rangle_{\mathrm{Sob}}-\langle K u, v\rangle_{\mathrm{Sob}} \\
& =\oint_{\partial \Sigma} d S_{i} N\left(\tilde{h}^{-1}\right)^{i j}\left[\left(D_{j} u\right)^{*}\left\{\left(q^{2}+K\right) v\right\}-\left\{\left(q^{2}+K\right) u\right\}^{*}\left(D_{j} v\right)\right]=0 .
\end{aligned}
$$

\section{THE EVAPORATION PROCESS ON NS1-W SOLUTION}

The metric of the target spacetime and the dilation field for the NS1-W solution [9] are given by

$$
\begin{aligned}
& d s_{d+1}^{2}=-\frac{f(r)}{H_{\alpha}(r) H_{\beta}(r)} d t^{2}+f^{-1}(r) d r^{2}+r^{2} d \Omega_{d-2}+\frac{H_{\beta}(r)}{H_{\alpha}(r)}\left(d X^{d}+\hat{A}_{\mu}(r) d x^{\mu}\right)^{2}, \\
& e^{-2 \Phi}=H_{\alpha}(r)=1+\left(\frac{r_{0}}{r}\right)^{d-3} \sinh ^{2} \alpha,
\end{aligned}
$$

where $f(r)=1-\left(r_{0} / r\right)^{d-3}$. The various quantities are summarized in the appendix B.

We denote the charges related to the Kalb-Ramond field $\mathcal{A}_{\mu}$ and to the KK U(1) field $\hat{A}_{\mu}$ by $Q_{\alpha}$ and $Q_{\beta}$, respectively. The extremal limit is that the horizon position $r_{0} \rightarrow 0, \alpha, \beta \rightarrow \infty$ keeping $Q_{\alpha}, Q_{\beta}$ fixed. The ADM mass $M$ is bounded below $M \geq Q_{\alpha}+Q_{\beta}$. 
The Hawking temperature and the entropy in the extremal limit are given by

$$
S_{\mathrm{BH}} \sim r_{0} \sqrt{Q_{\alpha} Q_{\beta}}, \quad T_{\mathrm{BH}} \sim \frac{r_{0}^{d-4}}{\sqrt{Q_{\alpha} Q_{\beta}}}
$$

so that, in the extremal limit, the temperature approaches a finite value $T_{H} \sim 1 / \sqrt{Q_{\alpha} Q_{\beta}}$ for $d=4$ and the zero temperature for $d \geq 5$. Therefore, it is very interesting to consider the evaporation process of near extremal five dimensional $(d=4)$ NS1-W solution.

In the background (III.23)-(III.24), the metric $\tilde{g}_{\mu \nu}$ appeared on the first quantization of the fundamental string is given by

$$
\begin{aligned}
& \tilde{g}_{\mu \nu} d x^{\mu} d x^{\nu}=-\frac{f(r)}{H_{\alpha}(r) H_{\beta}(r)} d t^{2}+\tilde{f}^{-1}(r) d r^{2}+r^{2} d \Omega_{d-2}, \\
& \tilde{f}(r):=f(r)\left[1+\frac{\gamma \alpha^{\prime}}{4} f\left(\frac{H_{\alpha}^{\prime}}{H_{\alpha}}\right)^{2}\right]^{-1},
\end{aligned}
$$

and the KK-compactified test string couples to $U(1)$ gauge field $\mathbb{A}_{\mu}$ and the potential $V$,

$$
\begin{aligned}
& \mathbb{A}_{\mu} d x^{\mu}=-\left((\kappa m) \frac{\sinh \alpha \cosh \alpha}{H_{\alpha}(r)}+q \frac{\sinh \beta \cosh \beta}{H_{\beta}(r)}\right)\left(\frac{r_{0}}{r}\right)^{d-3} d t, \\
& V=(\kappa m)^{2} \frac{H_{\beta}(r)}{H_{\alpha}(r)}+q^{2} \frac{H_{\alpha}(r)}{H_{\beta}(r)},
\end{aligned}
$$

where a dash means the derivative with respect to $r$.

As shown in the previous section, the only difference between usual complex scalar field equation and our effective field equation (II.14) derived from the first quantization is the difference between $g$ and $\tilde{g}$ appeared in the kinetic terms. Since $\sqrt{\alpha^{\prime}}$ is the string scale, the corrections from the dilaton field are negligibly small when the the size of the black hole $r_{0}$ is much larger than the string scale. However, they are important when the horizon becomes comparable to the string scale.

Anyway, since the background is static and spherically symmetric, for simplicity, we consider spherically symmetric solution (S-wave solution) $\Psi=e^{-i \omega t} \psi(r) / r^{(d-2) / 2}$, and then, Eq.(II.14) reduces to the radial equation

$$
\begin{aligned}
& 0=\left(\frac{d^{2}}{d \tilde{r}_{*}^{2}}+\frac{\tilde{f}}{f}\left(\omega+\mathbb{A}_{t}\right)^{2}-\tilde{f} U(r)\right) \psi, \\
& U:=\left(\frac{\kappa m}{H_{\alpha}}\right)^{2}+\left(\frac{q}{H_{\beta}}\right)^{2}+\frac{d-2}{2 r} \frac{1}{r^{(d-4) / 2}} \frac{1}{\sqrt{H_{\alpha} H_{\beta}}}\left(\frac{r^{(d-4) / 2} \tilde{f}}{\sqrt{H_{\alpha} H_{\beta}}}\right)^{\prime},
\end{aligned}
$$

where we introduce the modified tortoise coordinate $\tilde{r}_{*}$

$$
\tilde{r}_{*}:=\int d r \sqrt{H_{\alpha} H_{\beta}} / \tilde{f}
$$




\section{A. evaporation of near extremal black string with finite temperature}

We shall consider the evaporation of the five-dimensional NS1-W solution, which has finite temperature in the extremal limit.

First we investigate the process by the non-critical string model, $\gamma<0 \ddagger$. As the black string approaches the extremal state, $H_{\alpha}$ and $\tilde{f}$ can be approximated as

$$
H_{\alpha} \sim \frac{r_{0}}{r} \sinh ^{2} \alpha, \quad \tilde{f}=f\left(1+\frac{\gamma \alpha^{\prime} f}{4 r^{2}}\right)^{-1}
$$

near the horizon. When the size of the black hole becomes $r_{0}=(1 / 3) \sqrt{|\gamma| \alpha^{\prime} / 3}$, the effective field equation (III.30) is singular at $r_{1}=(1 / 2) \sqrt{|\gamma| \alpha^{\prime} / 3}$, since $\tilde{f}$ diverges at $r_{1}$ as $\tilde{f} \sim 1 /\left(r-r_{1}\right)^{2}$.

Let us consider a boundary condition on it. As a physically reasonable condition, we shall impose that the total energy $E$ is finite:

$$
E:=\int d \Sigma n_{\mu} \xi_{\nu} T^{\mu \nu}=\int d \Sigma N\left[\frac{\left|\mathcal{D}_{t} \Psi\right|^{2}}{N^{2}}+\tilde{h}^{i j}\left(D_{i} \Psi\right)^{*}\left(D_{j} \Psi\right)+V|\Psi|^{2}\right]<\infty
$$

where the energy-momentum tensor is usually defined by $T^{\mu \nu}:=(2 / \sqrt{-g})\left(\delta S_{\Psi} / \delta g_{\mu \nu}\right)$. Since $\tilde{h}^{i j} \sim \tilde{f}$, the above condition implies that for $r \sim r_{1}$,

$$
\Psi \sim\left(r-r_{1}\right)^{n}, \quad n>3 / 2
$$

Near the singularity, the wave-equation (III.30) is approximately written by

$$
0=\left(\frac{d^{2}}{d \tilde{r}_{*}^{2}}+c \tilde{r}_{*}^{-5 / 3}\right) \psi(r), \quad \text { for } \quad r>r_{1}
$$

where $c$ is a positive number and $r_{*} \sim\left(r-r_{1}\right)^{3}$. The two-independent solutions are expressed by Bessel functions:

$$
\begin{aligned}
& \psi_{1} \sim \sqrt{\tilde{r}_{*}} J_{3}\left(6 \sqrt{c} \tilde{r}_{*}^{1 / 6}\right) \sim \tilde{r}_{*} \sim\left(r-r_{1}\right)^{3}, \\
& \psi_{2} \sim \sqrt{\tilde{r}_{*}} N_{3}\left(6 \sqrt{c} \tilde{r}_{*}^{1 / 6}\right) \sim 1+a \tilde{r}_{*}^{1 / 3} \sim 1+a\left(r-r_{1}\right),
\end{aligned}
$$

where $a$ is a number. So, $E$ is finite for $\psi_{1}$, while $E$ diverges for $\psi_{2}$. This implies that the only $\psi_{1}$ mode is admitted for the singular boundary. Using Eq. (II.28), one can easily check that $\psi_{1}$ produces no string current on the boundary:

\footnotetext{
${ }^{\ddagger}$ Strictly speaking, we need to use a non-critical black string solution instead of the NS1-W solution for consistency with the non-critical string model. For our best knowledge, the non-critical black string solution is not yet obtained. So, we simply use the NS1-W solution to investigate the creation of the non-critical strings.
} 


$$
n_{\mu} J^{\mu} \sim \tilde{f} \psi_{1} \partial_{r} \psi_{1} \sim\left(r-r_{1}\right)^{3} \rightarrow 0
$$

So, the surface at $r=r_{1}$ plays a role of a reflective mirror. This means that the evaporation process ceases when the horizon reaches the string scale and that no naked singularity appears for the non-critical string case ${ }^{\S}$.

It is worthwhile to comment another meaning of the condition (III.34). Because $\langle\Psi, \Psi\rangle_{\text {Sob }}<$ (const.) $\times E<\infty$, the condition (III.34) imposes that the allowed states belong to the Sobolev space $H^{1}$ with the inner product (II.21). It is easy to check that $\psi_{1} \in H^{1}$ and $\psi_{2} \notin H^{1}$. This means that the operator $K$ is essentially self-adjoint in the Sobolev space, so that the initial value problem of $\Psi$ is well-posed [10] ${ }^{* *}$. Essential self-adjointness of $K$ is proved by showing that we have no solution in $H^{1}$ to the each eigenvalue equation $K \Psi= \pm i \Psi$ [11], which is reduced to the similar equation to Eq.(III.30),

$$
0=\left(\frac{d^{2}}{d \tilde{r}_{*}^{2}} \pm i \frac{\tilde{f}}{f}-\tilde{f} U(r)\right) \psi .
$$

Due to the singular behavior of the last term of $U$ near $r \sim r_{1}$, both equations become Eq.(III.36) and the solutions behave as Eq.(III.37), so that the solutions in $H^{1}$ of Eq.(III.39) should satisfy the boundary condition $\Psi\left(r=r_{1}\right)=\Psi\left(r_{*}=0\right)=0$ and also $\Psi\left(r_{*}=\infty\right)=0$. We will have no solution of the eigenvalue equation (III.39) satisfying the above boundary condition, because we have no room to adjust the eigenvalues. Thus, the operator $K$ will be essentially self-adjoint in the Sobolev space.

Secondly, we consider the evaporation process by the critical string model, $\gamma>0$. As easily checked, the effective potential $U$ in Eq. (III.31) is bounded above by the charges $Q_{\alpha}$ and $Q_{\beta}$ as

$$
U \lesssim 1 / Q_{\alpha} Q_{\beta}
$$

in the extremal limit, implying that the evaporation continues until naked singularity appears in the extremal limit. What has to be noticed is that we implicitly assumed that the NS1-W black

\footnotetext{
${ }^{\S}$ When the size of the black string becomes $r_{0}<(1 / 3) \sqrt{|\gamma| \alpha^{\prime} / 3}, \tilde{f}$ can be negative in the region $r \in\left(r^{\prime}, r^{\prime \prime}\right)$ outside the horizon, where $r_{0}<r^{\prime}<r_{1}<r^{\prime}$. So, we have "two time" $\tilde{g}_{t t}, \tilde{g}_{r r}<0$ in the region. However, by the same argument in the main text, we can show that the surface at $r=r^{\prime \prime}$ also plays a role of a reflective mirror owing to the behavior of $\tilde{f}$ at $r=r^{\prime \prime}$ as $\tilde{f} \sim 1 /\left(r-r^{\prime \prime}\right)$ and it makes the "two time" region separate from the asymptotic region $r^{\prime \prime}<r$.

${ }^{* *}$ On the other hand, by the method similar to that noted below, we can show that the operator $K$ is not essentially self-adjoint in the linear function space with the square integrability $L^{2}$, so that the evolution is not unique.
} 
string eventually evaporates and then the quasi-adiabatic approximation is valid for all time. We will argue that this condition is violated at the final stage of the evaporation and that a timelike mirror surface would be formed even in the critical string model in section IV.

\section{B. evaporation of the near extremal black string solution with zero temperature}

In this subsection, we discuss whether or not the black string solution in which temperature is zero in the extremal limit is stable against the string creations. Since the string creation caused by gravitational effect is negligibly small compared with the superradiance or the spontaneous discharge near the extremal limit, the stability is determined by the spontaneous discharge process only.

The condition for the spontaneous discharge is that there exist the modes with negative phase velocity. From the wave equation (III.30) near the horizon

$$
0=\left(\frac{d^{2}}{d \tilde{r}_{*}^{2}}+\Omega_{\omega}^{2}+O(f)\right) \psi_{\omega \lambda}, \quad \Omega_{\omega}:=\omega-[(\kappa m) \tanh \alpha+q \tanh \beta],
$$

they should satisfy

$$
\omega<(\kappa m) \tanh \alpha+q \tanh \beta=: \omega_{\mathrm{SR}} .
$$

From the wave equation near infinity

$$
0=\left(\frac{d^{2}}{d r^{2}}+\tilde{\Omega}_{\omega}^{2}+O\left(r^{-2}\right)\right) \psi_{\omega \lambda}, \quad \tilde{\Omega}_{\omega}:=\sqrt{\omega^{2}-\left[(\kappa m)^{2}+q^{2}\right]},
$$

the modes should also satisfy

$$
\omega^{2}>(\kappa m)^{2}+q^{2}=: \mu^{2}
$$

for the wave propagation to infinity. Combining the above two inequalities, we obtain the stability condition against the spontaneous discharge process, $\mu>\omega_{\mathrm{SR}}$, which is automatically satisfied by the BPS-like bound $\mu>(\kappa m)+q \geq \omega_{\mathrm{SR}}$ and is equivalent to

$$
\frac{(\kappa m)^{2}}{\cosh ^{2} \alpha}+\frac{q^{2}}{\cosh ^{2} \beta}>2(\kappa m) q \tanh \alpha \tanh \beta .
$$

So, provided that this condition (III.45) holds, the extremal black string solution with zero temperature is stable against the string creation.

In the extremal limit $\alpha, \beta \rightarrow \infty$, the condition (III.45) is automatically broken unless $q=0$, so that the black string solution with the $\mathrm{W}$-charge is unstable. By the standard procedure [8], the 
mass-loss rate and the charge-loss become

$$
\begin{aligned}
& \frac{d M}{d t}=-\int_{\mu}^{\omega_{\mathrm{SR}}} d \omega \omega\left(\left|R_{\omega}\right|^{2}-1\right), \\
& \frac{d Q_{\alpha}}{d t}=-(\kappa m) \int_{\mu}^{\omega_{\mathrm{SR}}} d \omega\left(\left|R_{\omega}\right|^{2}-1\right), \quad \frac{d Q_{\beta}}{d t}=-q \int_{\mu}^{\omega_{\mathrm{SR}}} d \omega\left(\left|R_{\omega}\right|^{2}-1\right),
\end{aligned}
$$

where $R_{\omega}$ is the reflection amplitude and for the superradiance modes (III.42), $\left|R_{\omega}\right|^{2}>1$.

Since $\kappa m+q \geq \omega_{\mathrm{SR}}$ and $\left|R_{\omega}\right|^{2}>1$ for $\omega_{\mathrm{SR}}>\omega$, we conclude that

$$
0>\frac{d M}{d t}>\frac{d Q_{\alpha}}{d t}+\frac{d Q_{\beta}}{d t} \quad \Longrightarrow \quad\left|\frac{d M}{d t}\right|<\left|\frac{d}{d t}\left(Q_{\beta}+Q_{\beta}\right)\right| .
$$

This means that the near-extremal black string has more discharge rate than mass-loss one. Therefore provided that the quasi-adiabatic approximation is valid, the over-charge process does not occur even for the black string solution with the W-charge, so that the naked singularity at the extremal state does not appear for the black string solution with zero temperature in both the critical and non-critical string models.

\section{CONCLUSION AND DISCUSSIONS}

We investigated the effect of dilaton field on string creation in near-extremal NS1-W black string solutions. For a simplicity we assumed that the string extends along one Killing direction only. So, it is reduced to a charged particle whose motion is derived from the effective action (II.10) in the lower dimensional spacetime.

As shown in Sec.III A the determinant of the "effective metric" $\tilde{g}_{\mu \nu}$ becomes zero at a timelike surface outside the horizon when the horizon size is close to the Planck scale in the non-critical string model. It follows that the field equation describing the particle creation becomes singular at the surface. Assuming that all the states belong to the Sobolev space $H^{1}$, we showed that the evolution is uniquely defined for all time and that there is no particle flux propagating across the surface. In other words, the black string at the Planck scale is completely enclosed by the timelike "mirror" surface and hence the evaporation by the Hawking radiation stops. Of course, this conclusion is based on perturbative treatment of strings and the quasi-adiabatic approximation. Although we need any non-perturbative treatment of string theories for a complete understanding of the whole evaporation process due to the formation of the mirror surface at the Planck regime, it is beyond our scope.

It is worth noting that we used a five-dimensional NS1 black string solution as a background spacetime instead of a non-critical solution simply because no analytical black hole solution is found 
in the non-critical string theory. We expect, however, that the determinant of the "effective metric" $\tilde{g}_{\mu \nu}$ becomes zero near curvature singularity even in the non-critical solution unless the dilaton field is finite there.

Finally we comment on the case for the critical string model in a five-dimensional NS1 black string solution under the quasi-adiabatic approximation. Since the timelike mirror surface is not produced in this case, the evaporation by the Hawking radiation seems continue until the naked singularity appears. Let us consider if the surface is produced or not when we take into account the time-dependence of the dilaton field. As easily seen in Eq. (III.24), the dilaton field diverges at the extremal state. So, the time derivative would also diverge at the extremal state if the evaporation is completed within a finite time. Since the time component of the metric $\tilde{g}$ changes as

$$
\tilde{g}_{t t}=g_{t t}+\gamma\left(\partial_{t} \Phi\right)^{2}
$$

it would become zero at the last stage (note that $\gamma>0$ in the critical string model). This implies that the a mirror surface preventing the formation of the naked singularity is produced provided that the time dependence is taken into account. This will be discussed in more detail in [12].

\section{ACKNOWLEDGMENTS}

It is a pleasure to thank M. Natsuume and S. Yahikozawa for continuous discussions.

\section{APPENDIX A: DERIVATION OF THE MODEL ACTION (II.2)}

We consider the bosonic string in the background fields, $G_{\mu \nu}(X), B_{\mu \nu}(X)$ and $\Phi(X)$, whose action is given by ${ }^{\dagger \dagger}$

$$
\begin{gathered}
S[X, h ; G, B, \Phi]=\frac{1}{4 \pi \alpha^{\prime}} \int d^{2} \sigma \sqrt{h}\left[\left\{h^{a b} G_{\mu \nu}(X)+i \epsilon^{a b} B_{\mu \nu}(X)\right\} \partial_{a} X^{\mu} \partial_{b} X^{\nu}\right. \\
\left.+\alpha^{\prime} R \Phi(X)\right] .
\end{gathered}
$$

We define the partition function for the bosonic string as

$$
Z[G, B, \Phi]:=\int \frac{D_{h} h D_{h} X}{V_{\text {diffeo. }} \times V_{\text {Weyl }}} e^{-S[X, h ; G, B, \Phi]},
$$

\footnotetext{
${ }^{\dagger \dagger}$ We follow the sign convention of the Polchinski's textbook [13].
} 
where $D_{h} \bullet$ means that it is invariant measure with respect to the world sheet metric $h_{a b}{ }^{\ddagger}$. By infinitesimal diffeomorphic $\left(\delta \xi_{a}\right)$ and Weyl transformations $(\delta \omega)$, the metric changes into

$$
\delta h_{a b}=2 \delta \omega h_{a b}-2 \nabla_{(a} \delta \xi_{b)},
$$

and the volumes of the diffeomorphism and the Weyl transformation are given by $V_{\text {diffeo. }}=\int D_{h} \xi$ and $V_{\mathrm{Weyl}}=\int D_{h} \omega$, respectively.

According to the standard procedure, we try to rewrite Eq.(A.2) using

$$
1=\Delta_{\mathrm{FP}}[h] \int D_{h} \zeta \delta\left[h-\hat{h}^{\zeta}\right],
$$

where $\hat{h}_{a b}$ is a fiducial metric and $\zeta$ means diffeomorphic $\left(\xi_{a}\right)$ and Weyl transformation $(\omega)$ parameters, so that $D_{h} \zeta=D_{h} \omega \times D_{h} \xi$. From Eqs.(A.2) and (A.4),

$$
\begin{aligned}
Z[G, B, \Phi] & =\int \frac{D_{h} h D_{h} X D_{h} \zeta}{V_{\text {diffeo. }} \times V_{\text {Weyl }}} \Delta_{\mathrm{FP}}[h] \delta\left[h-\hat{h}^{\zeta}\right] e^{-S[X, h ; G, B, \Phi]} \\
& =\int \frac{D_{\hat{h}^{\zeta}} X D_{\hat{h}^{\zeta}} \zeta}{V_{\text {diffeo. }} \times V_{\text {Weyl }}} \Delta_{\mathrm{FP}}\left[\hat{h}^{\zeta}\right] e^{-S\left[X, \hat{h}^{\zeta} ; G, B, \Phi\right]}
\end{aligned}
$$

and from the fact that various quantities are obviously the world sheet diffeomorphic invariant ones, the partition function becomes

$$
\begin{aligned}
& Z[G, B, \Phi]=\int \frac{D_{\hat{h}^{\omega}} X D_{\hat{h}^{\omega}} \omega D_{\hat{h}^{\omega}} \xi}{V_{\text {diffeo. }} \times V_{\text {Weyl }}} \Delta_{\mathrm{FP}}\left[\hat{h}^{\omega}\right] e^{-S\left[X, \hat{h}^{\omega} ; G, B, \Phi\right]} \\
& =\int \frac{D_{\hat{h}^{\omega}} X D_{\hat{h}^{\omega} \omega} \omega}{V_{\mathrm{Weyl}}} \Delta_{\mathrm{FP}}\left[\hat{h}^{\omega}\right] e^{-S\left[X, \hat{h}^{\omega} ; G, B, \Phi\right]},
\end{aligned}
$$

where $\hat{h}_{a b}^{\omega}:=e^{2 \omega} \hat{h}_{a b}$ and we use $\int D_{\hat{h}^{\omega}} \xi=V_{\text {diffeo. }}$ Furthermore, Eq.(A.6) can be rewritten as

$$
Z[G, B, \Phi]=\int \frac{D_{\hat{h}} X D_{\hat{h}} \omega}{V_{\mathrm{Weyl}}} \Delta_{\mathrm{FP}}[\hat{h}] \times \frac{D_{\hat{h}^{\omega}} \omega}{D_{\hat{h}} \omega} \frac{D_{\hat{h}^{\omega}} X}{D_{\hat{h}} X} \frac{\Delta_{\mathrm{FP}}\left[\hat{h}^{\omega}\right]}{\Delta_{\mathrm{FP}}[\hat{h}]} e^{-S\left[X, \hat{h}^{\omega} ; G, B, \Phi\right]},
$$

and the Jacobian of the functional volume element produces the Liouville action

$$
\begin{aligned}
& \frac{D_{\hat{h}^{\omega}} \omega}{D_{\hat{h}} \omega} \frac{D_{\hat{h}^{\omega}} X}{D_{\hat{h}} X} \frac{\Delta_{\mathrm{FP}}\left[\hat{h}^{\omega}\right]}{\Delta_{\mathrm{FP}}[\hat{h}]}=\exp \left(\frac{D-D_{\text {crit }}+1}{24 \pi} S_{L}[\omega, \hat{h}]\right), \\
& S_{L}[\omega, \hat{h}]:=\int d^{2} \sigma \sqrt{\hat{h}}(\omega \hat{R}-\omega \hat{\Delta} \omega),
\end{aligned}
$$

\footnotetext{
${ }^{\ddagger \ddagger}$ Moreover, $D_{h} X$ should also be invariant measure with respect to $G_{\mu \nu}$.
} 
where $D_{\text {crit }}=26$ is the critical dimension for the bosonic string and $\hat{\Delta}$ is the Laplace-Beltrami operator with respect to $\hat{h}$. Thus, we obtain

$$
\begin{aligned}
& Z[G, B, \Phi]=\int \frac{D_{\hat{h}} X D_{\hat{h}} \omega}{V_{\mathrm{Weyl}}} \Delta_{\mathrm{FP}}[\hat{h}] e^{-I[X, \omega, \hat{h} ; G, B, \Phi]} \\
& I[X, \omega, \hat{h} ; G, B, \Phi]:=S\left[X, \hat{h}^{\omega} ; G, B, \Phi\right]-\frac{D-D_{\text {crit }}+1}{24 \pi} S_{L}[\omega, \hat{h}] .
\end{aligned}
$$

By making use of the equation $\sqrt{\hat{h}^{\omega}} \hat{R}\left[\hat{h}^{\omega}\right]=\sqrt{\hat{h}}(\hat{R}-2 \hat{\Delta} \omega)$ and gathering $\omega$-dependence, we can rewrite Eq.(A.11) as

$$
I[X, \omega, \hat{h} ; G, B, \Phi]=\mathcal{S}[X, \hat{h} ; G, B, \Phi]-\frac{D-D_{\text {crit }}+1}{24 \pi} S_{L}\left[\omega^{\prime}, \hat{h}\right],
$$

where $\omega^{\prime}:=\omega-6 \Phi /\left(D-D_{\text {crit }}+1\right)$. And then, $\mathcal{S}$ is given by

$$
\begin{aligned}
& \mathcal{S}:=\frac{1}{4 \pi \alpha^{\prime}} \int d^{2} \sigma \sqrt{\hat{h}}\left(\hat{h}^{a b} \tilde{G}_{M N}(X)+\hat{\epsilon}^{a b} B_{M N}(X)\right) \partial_{a} X^{M} \partial_{b} X^{N}, \\
& \tilde{G}_{M N}(X):=G_{M N}(X)+\gamma \alpha^{\prime} \partial_{M} \Phi(X) \cdot \partial_{N} \Phi(X)
\end{aligned}
$$

where $\gamma:=6 /\left(D-D_{\text {crit }}+1\right)$.

Finally, we can equivalently rewrite Eq.(A.10) in the factored form

$$
\begin{aligned}
Z[G, B, \Phi] & =\int \frac{D_{\hat{h}} \omega^{\prime}}{V_{\mathrm{Weyl}}} e^{\frac{D-D_{\text {crit }}+1}{24 \pi} S_{L}\left[\omega^{\prime}, \hat{h}\right]} \times \int D_{\hat{h}} X \Delta_{\mathrm{FP}}[\hat{h}] e^{-\mathcal{S}[X, \hat{h} ; G, B, \Phi]} \\
& =\int \frac{D_{\hat{h} \omega} \omega}{V_{\mathrm{Weyl}}} e^{\frac{D-D_{\text {crit }}}{24 \pi} S_{L}[\omega, \hat{h}]} \times \int D_{\hat{h}} X \Delta_{\mathrm{FP}}[\hat{h}] e^{-\mathcal{S}[X, \hat{h} ; G, B, \Phi]}
\end{aligned}
$$

where we change $\omega^{\prime} \rightarrow \omega$ and the path integral measure, $D_{\hat{h}} \omega \rightarrow D_{\hat{h}} \omega \omega$ in the last equality. Thus, the physical modes $X$ decouple from the conformal mode of the world sheet metric $\omega$, so that we can treat the coupling between a test string and the dilaton in the conformal invariant manner (A.13).

Obviously from the above procedure, the action (A.13) is also applicable for the superstring by replacing $D_{\text {crit }}=26$ with $D_{\text {crit }}=10$, so that we have $\gamma=6 /(D-9)$ for the superstring.

\section{APPENDIX B: NS1-W SOLUTION}

In this appendix, we summarize the NS1-W solution. The metric of the $(d+1)$-dimensional target spacetime for the NS1-W solution [9] is given by

$$
\begin{aligned}
& d s_{d+1}^{2}=-\frac{f(r)}{H_{\alpha}(r) H_{\beta}(r)} d t^{2}+f^{-1}(r) d r^{2}+r^{2} d \Omega_{d-2}+\frac{H_{\beta}(r)}{H_{\alpha}(r)}\left(d X^{d}+\hat{A}_{\mu}(r) d x^{\mu}\right)^{2}, \\
& f(r)=1-\left(\frac{r_{0}}{r}\right)^{d-3}, \quad \hat{A}_{\mu}(r) d x^{\mu}=-\left(\frac{r_{0}}{r}\right)^{d-3} \frac{\sinh \beta \cosh \beta}{H_{\beta}(r)} d t
\end{aligned}
$$


where

$$
H_{\alpha}(r)=1+\left(\frac{r_{0}}{r}\right)^{d-3} \sinh ^{2} \alpha, \quad H_{\beta}(r)=1+\left(\frac{r_{0}}{r}\right)^{d-3} \sinh ^{2} \beta .
$$

The Kalb-Ramond field and the dilaton field are given by

$$
\boldsymbol{B}=\mathcal{A}_{\mu} d x^{\mu} \wedge d X^{d}=\left[-\left(\frac{r_{0}}{r}\right)^{d-3} \frac{\sinh \alpha \cosh \alpha}{H_{\alpha}(r)} d t\right] \wedge d X^{d}, \quad e^{-2 \Phi}=H_{\alpha}(r),
$$

and, the charges of $\mathcal{A}_{\mu}$ related to the Kalb-Ramond field and the KK U(1) field $\hat{A}_{\mu}$ are defined by

$$
Q_{\alpha}:=\frac{1}{16 \pi G_{d}} \int_{S_{d-2}^{\infty}} \frac{e^{-2 \phi}}{k^{2}} * \mathcal{F}, \quad Q_{\beta}:=\frac{1}{16 \pi G_{d}} \int_{S_{d-2}^{\infty}} k^{2} e^{-2 \phi *} \hat{\boldsymbol{F}}
$$

respectively. Where $G_{d}$ is the $d$-dimensional Newton constant related to the $(d+1)$-dimensional Newton constant $G_{d+1}$ as $G_{d}=G_{d+1} / l_{z}$, and the field $\phi$ is the $d$-dimensional dilaton field given by $\phi=\Phi-(1 / 2) \ln k$. Another important conserved quantity is the ADM mass and it is easily read off in the Einstein frame $g_{\mu \nu}^{(E)}:=e^{-4 \phi /(d-2)} g_{\mu \nu}$ as

$$
g_{t t}^{(E)} \sim-1+\frac{16 \pi G_{d}}{(d-2) V_{d-2}} \frac{M}{r^{d-3}}+\cdots .
$$

From Eqs.(B.5) and (B.6), we have for the NS1-W solution

$$
\begin{aligned}
M & =\frac{l_{z} V_{d-2}}{16 \pi G_{d+1}} r_{0}^{d-3}\left[d-2+(d-3)\left(\sinh ^{2} \alpha+\sinh ^{2} \beta\right)\right], \\
Q_{\alpha} & =(d-3) \frac{l_{z} V_{d-2}}{16 \pi G_{d+1}} r_{0}^{d-3} \sinh \alpha \cosh \alpha, \\
Q_{\beta} & =(d-3) \frac{l_{z} V_{d-2}}{16 \pi G_{d+1}} r_{0}^{d-3} \sinh \beta \cosh \beta .
\end{aligned}
$$

Furthermore, the Hawking temperature and the entropy of the $\mathrm{BH}$ are given by

$$
S_{\mathrm{BH}}=\frac{l_{z} V_{d-2}}{4 G_{d+1}} r_{0}^{d-2} \cosh \alpha \cosh \beta, \quad T_{\mathrm{H}}=\frac{d-3}{4 \pi r_{0} \cosh \alpha \cosh \beta} .
$$

[1] L. J. Dixon, J. A. Harvey, C. Vafa, and E. Witten, Nucl. Phys. B. 261 (1985) 678

[2] G. T. Horowitz and A. R. Steif, Phys. Rev. Lett. 64 (1990) 260, Phys. Rev. D42 (1990) 1950

[3] A. Lawrence and E. Martinec, Class. Quantum. Grav. 13 (1996) 63

[4] J. J. Friess, S. S. Gubser, and I. Mitra, Nucl. Phys. B. 689 (2004) 243

[5] S. S. Gubser, Phys. Rev. D69 (2004) 123507

[6] J. Koga and K. Maeda, Phys. Rev. D52 (1995) 7066

[7] A. Ishibashi and A. Hosoya, Phys. Rev. D60 (1999) 104028 
[8] G.W. Gibbons, Comm. Math. Phys. 44, 245 (1975).

[9] A. Dabholkar, G.W. Gibbons, J. Harvey and F. Ruiz-Ruiz, Nucl. Phys. B340, 33 (1990); A. Dabholkar and J. Harvey, Phys. Rev. Lett. 63, 478 (1989); M. J. Duff, R.R. Khuri and J.X. Lu, Phys. Rep. 259, 213 (1995).

[10] S.W. Hawking and G.F.R. Ellis, Large Scale Structure of Spacetimes (Cambridge University Press, Cambridge, England, 1973).

[11] M. Reed and B. Simon, Fourier Analysis, Self-Adjointness (Academic Press, New York, 1975).

[12] K. Maeda and T. Okamura, in preperation.

[13] J. Polchinski, String theory (Cambridge University Press, Cambridge, 1998). 